\title{
SPARC Aggravates Blood-Brain Barrier Disruption via Integrin $\alpha$ V $\beta$ 3/MAPKs/MMP-9 Signaling Pathway after Subarachnoid Hemorrhage
}

\author{
Takeshi Okada $\mathbb{D},{ }^{1,2,3}$ Hidenori Suzuki $\mathbb{D}^{2}$ Zachary D. Travis $\mathbb{D}^{3},{ }^{3,4}$ Orhan Altay $\mathbb{D}^{3}{ }^{3}$ \\ Jiping Tang $\oplus^{3}$, and John H. Zhang $\oplus^{3,5,6}$ \\ ${ }^{1}$ Department of Neurosurgery, Kuwana City Medical Center, 3-11 Kotobuki-cho, Kuwana, Mie 511-0061, Japan \\ ${ }^{2}$ Department of Neurosurgery, Mie University Graduate School of Medicine, 2-174 Edobashi, Tsu, Mie 514-8507, Japan \\ ${ }^{3}$ Department of Physiology and Pharmacology, Loma Linda University, Risley Hall, Room 219, 11041 Campus St., Loma Linda, \\ CA 92354, USA \\ ${ }^{4}$ Department of Earth and Biological Sciences, Loma Linda University, Risley Hall, Room 219, 11041 Campus St., Loma Linda, \\ CA 92354, USA \\ ${ }^{5}$ Department of Anesthesiology, Loma Linda University, Risley Hall, Room 219, 11041 Campus St., Loma Linda, CA 92354, USA \\ ${ }^{6}$ Department of Neurosurgery, Loma Linda University, Risley Hall, Room 219, 11041 Campus St., Loma Linda, CA 92354, USA
}

Correspondence should be addressed to John H. Zhang; johnzhang3910@yahoo.com

Received 20 August 2021; Accepted 18 October 2021; Published 11 November 2021

Academic Editor: Alin Ciobica

Copyright (C) 2021 Takeshi Okada et al. This is an open access article distributed under the Creative Commons Attribution License, which permits unrestricted use, distribution, and reproduction in any medium, provided the original work is properly cited.

\begin{abstract}
Blood-brain barrier (BBB) disruption is a common and critical pathology following subarachnoid hemorrhage (SAH). We investigated the BBB disruption property of secreted protein acidic and rich in cysteine (SPARC) after SAH. A total of 197 rats underwent endovascular perforation to induce SAH or sham operation. Small interfering ribonucleic acid (siRNA) for SPARC or scrambled siRNA was administered intracerebroventricularly to rats $48 \mathrm{~h}$ before SAH. Anti-SPARC monoclonal antibody (mAb) 236 for functional blocking or normal mouse immunoglobulin G (IgG) was administered intracerebroventricularly $1 \mathrm{~h}$ after SAH. Selective integrin $\alpha \mathrm{V} \beta 3$ inhibitor cyclo(-RGDfK) or phosphate-buffered saline was administered intranasally $1 \mathrm{~h}$ before SAH, along with recombinant SPARC treatment. Neurobehavior, SAH severity, brain edema, immunohistochemical staining, and Western blot were evaluated. The expression of SPARC and integrin $\alpha \mathrm{V} \beta 3$ was upregulated after SAH in the endothelial cells. SPARC siRNA and anti-SPARC mAb 236 prevented neuroimpairments and brain edema through protection of $\mathrm{BBB}$ as measured by IgG extravasation 24 and $72 \mathrm{~h}$ after SAH. Recombinant SPARC aggravated neuroimpairments and cyclo(-RGDfK) suppressed the harmful neurological effects via inhibition of activated c-Jun N-terminal kinase, p38, and matrix metalloproteinase-9 followed by retention of endothelial junction proteins. SPARC may induce post-SAH BBB disruption via integrin $\alpha \mathrm{V} \beta 3$ signaling pathway.
\end{abstract}

\section{Introduction}

Aneurysmal subarachnoid hemorrhage (SAH) is a serious life-threatening cerebrovascular disease arising from a ruptured intracranial aneurysm [1-3]. Following an aneurysm rupture, arterial blood spreads into the subarachnoid space leading to devastating brain injury. This brain injury is caused by blood components, their secondary products, which are induced by tissue damage, and mechanical stress caused by the rapid elevation of intracranial pressure [4-7]. Many secondary products induced by tissue damage have been reported to cause the breakdown of the endothelial junctions forming the blood-brain barrier (BBB), leading to vasogenic edema (which is a major cause of brain injury and an independent risk factor for mortality and poor outcome after SAH [4, 8]). Moreover, enhanced BBB 
permeability allows immune molecules to migrate into brain parenchyma, which further propagates brain injury [9]. Therefore, BBB disruption is a complication which needs to be addressed in order to improve clinical outcomes in SAH. Indeed, a number of papers suggest that treatment options to preserve endothelial junction proteins to protect $\mathrm{BBB}$ could be beneficial in improving neurological functions in experimental SAH models [10]. However, the molecular mechanisms of post-SAH BBB disruption remain unclear.

Secreted protein acidic and rich in cysteine (SPARC), also known as osteonectin and basement-membrane 40, is a type of matricellular proteins (MCPs). SPARC is a secondary product induced in response to injury and exerts diverse functions while the levels of protein expression are low in a steady-state condition, and knockouts of MCPs in mice undergo normal development $[11,12]$. SPARC may be involved in $\mathrm{BBB}$ disruption in the acute phase of $\mathrm{SAH}$ although it has never been investigated as to post-SAH brain injury including BBB disruption. The SPARC levels are elevated in microvascular blood vessels and astrocytes at sites of central nervous system injury [13-15]. An in vitro study demonstrated that exposure to SPARC increased vascular permeability and decreases expression of tight junction marker, zonula occludens (ZO)-1, and occludin [13].

Integrins are a superfamily of cell adhesion receptors that recognize mainly ECMs and cell-surface ligands and are composed of $\alpha$ and $\beta$ subunits forming 24 known combinations [16]. Of the integrin family, integrin $\alpha \mathrm{V} \beta 3$ is located in endothelial cells [17] and recognizes SPARC, activating the downstream signaling involving mitogenactivated protein kinases (MAPKs), proinflammatory mediators such as interleukins (ILs), and matrix metalloproteinase (MMP)-9 [16-24], although previous studies have never been investigated as to integrin $\alpha \mathrm{V} \beta 3$ signaling pathway in SAH. MMP-9 is a proteolytic enzyme to cause BBB disruption in SAH [4]. In addition, activated integrin $\alpha \mathrm{V} \beta 3$ induces ZO-1 and occludin internalization, disrupts vascular endothelial- (VE-) cadherin localization, and increased expression of MMP-9 [25]. Therefore, post-SAH SPARC induction may disrupt the BBB via MAPKs/MMP-9 signaling pathway. Thus, the aim of this study was to investigate if SPARC exerts BBB disruption in post-SAH and if SPARC signaling involves integrin $\alpha \mathrm{V} \beta 3$ and the downstream molecule MAPKs.

\section{Materials and Methods}

The Institutional Animal Care and Use Committee (IACUC) of Loma Linda University (LLU) approved the study protocol (No. 8170018), and all experiments were conducted in accordance with the NIH guidelines for the use of Animals in Neuroscience Research as well as the ARRIVE guidelines. All authors have read and approved the submitted manuscript.

2.1. Animal Model. Ten-week-old Sprague-Dawley male rats (weight, 300-320 g) were used to produce SAH models by endovascular perforation as previously described [5]. Briefly, each rat was intubated under deep anesthesia and main- tained by mechanical ventilation with $3 \%$ isoflurane, and a respiration rate of $77 / \mathrm{min}$ for the duration of the surgery. After exposing the left common, external, and internal carotid arteries, a sharpened 4-0 monofilament nylon suture was advanced rostrally into the left internal carotid artery from the external carotid artery stump to perforate the bifurcation of the anterior and the middle cerebral arteries. Sham-operated rats underwent identical procedures except that the suture was withdrawn without puncturing an artery. We monitored peak inspiration pressure and respiratory rate by a pressure-controlled ventilator, heart rate, skin pigmentation, and pedal reflex (firm toe pinch) during operation. These vitals were monitored every $5 \mathrm{~min}$ to ensure that animals were not in distress and were responding to the anesthesia and procedure accordingly. Body temperature was kept constant $37^{\circ} \mathrm{C}$ by a heating pad until the animals recovered. All rats were randomly assigned to the following experimental groups as described (Additional file 1: Figure S1). The time points were selected based on the previous studies, which reported that post-SAH BBB disruption peaked within $24 \mathrm{~h}$ and was reversed by $48-72 \mathrm{~h}$ following experimental SAH $[23,26]$. In the present study, the brain water content was measured at 24 and $72 \mathrm{~h}$ postsurgery, and Western blot (WB), except experiment 1, and immunohistochemical staining were performed at $24 \mathrm{~h}$ postsurgery in all experiments.

\subsection{Study Protocol}

2.2.1. Experiment 1. To determine the time course and cellular localization of endogenous SPARC and integrin $\alpha \mathrm{V} \beta 3$ expression, 40 rats were randomly divided and assigned to six groups: sham and SAH 3, 6, 12, 24, and $72 \mathrm{~h}$ groups. After mortality and SAH severity were evaluated, WB samples from the left hemisphere were analyzed $(n=6$ per group). Also, double immunohistochemical staining of SPARC and integrin $\alpha \mathrm{V} \beta 3$ in conjunction with astrocytes and cerebral endothelial cells was performed in the sham and SAH group at $24 \mathrm{~h}$ after modeling ( $n=2$ per group).

2.2.2. Experiment 2.1. To evaluate the effects of the knockdown of SPARC on post-SAH BBB disruption at $24 \mathrm{~h}, 48$ rats were randomly divided and assigned to four groups: sham $(n=14)$, SAH $(n=6), \mathrm{SAH}+$ scrambled small interfering ribonucleic acid (Scr siRNA; 500 pmol; $n=14$ ), and $\mathrm{SAH}+\mathrm{SPARC}$ siRNA (500 pmol; $n=14)$ groups. Negative control Scr siRNA (SR30004, OriGene Technologies, Rockville, MD) and SPARC siRNA (200133, Thermo Fisher Scientific Inc., Waltham, MA) were administered intracerebroventricularly (i.c.v) $48 \mathrm{~h}$ before modeling. Mortality, neurological scores, SAH severity, brain water content (BWC; $n=6$ per group), immunohistochemical staining $(n=4$ per group), and WB ( $n=4$ per group) were evaluated at $24 \mathrm{~h}$ after modeling.

2.2.3. Experiment 2.2. To evaluate the effects of the knockdown of SPARC on outcomes at $72 \mathrm{~h}, 18$ rats were randomly divided and assigned into three groups: sham, SAH + Scr siRNA, and SAH + SPARC siRNA (500 pmol) groups ( $n=6$ per group). Negative control Scr siRNA and SPARC 
siRNA were administered i.c.v $48 \mathrm{~h}$ before SAH induction. Mortality, neurological scores, SAH severity, and brain water content were evaluated at $72 \mathrm{~h}$ after modeling.

2.2.4. Experiment 3. To confirm the involvement of endogenous SPARC on post-SAH BBB disruption, 30 rats were randomly assigned to three groups: sham, $\mathrm{SAH}+$ normal mouse immunoglobulin G (IgG; $0.3 \mu \mathrm{g})$, SAH + SPARC monoclonal antibody $(\mathrm{mAb}) 236(0.3 \mu \mathrm{g})$ groups $(n=10$ per group). Negative control normal mouse IgG (12-371, EMD Millipore Inc., Temecula, CA) and functional blocking antibody SPARC mAb 236 (AB_2617208, Developmental Studies Hybridoma Bank, Iowa city, IA) were administered i.c.v $1 \mathrm{~h}$ after SAH induction. After mortality, neurological scores, and SAH severity were evaluated, brain water content ( $n=6$ per group) and immunohistochemical staining ( $n=4$ per group) were assessed $24 \mathrm{~h}$ after modeling.

2.2.5. Experiment 4. To investigate the effects of exogenous SPARC and the involvement of integrin $\alpha \mathrm{V} \beta 3$ on BBB disruption, 30 rats were randomly assigned to five groups: sham + phosphate-buffered saline (PBS), sham + recombinant human SPARC (rSPARC; $0.3 \mu \mathrm{g}$ ), SAH + PBS, SAH + rSPARC, and SAH + rSPARC + cyclo(-RGDfK) (cRGD; $2.0 \mu \mathrm{g}$ ) groups ( $n=6$ per group). A rSPARC (941-SP, R\&D Systems Inc., Minneapolis, MN) was administered i.c.v $1 \mathrm{~h}$ after modeling, and a selective integrin $\alpha \mathrm{V} \beta 3$ inhibitor cRGD (S7834, Selleckchem Inc., Houston, TX) was administered intranasally $1 \mathrm{~h}$ before SAH induction. After mortality, neurological scores, and SAH severity were evaluated, WB samples from the left hemisphere were analyzed $24 \mathrm{~h}$ after modeling.

2.3. SAH Severity and Exclusion Criteria. The severity of SAH was blindly assessed for each experimental animal that was sacrificed as previously described $[5,27]$. Briefly, the basal cistern was divided into 6 segments, and each segment was allotted a grade from 0 to 3 depending on the amount of $\mathrm{SAH}$. The rats received a total score ranging from 0 to 18 by adding the scores from all 6 segments. In the analysis, mild SAH (SAH grade $\leq 7$ ) rats were excluded, because mild $\mathrm{SAH}$ did not induce neurological impairments [5].

2.4. Intracerebroventricular Infusion. An i.c.v infusion was performed as previously described [5]. Briefly, rats were placed in a stereotaxic apparatus under $2-2.5 \%$ isoflurane anesthesia. The needle of a $10 \mu \mathrm{l}$ Hamilton syringe (Hamilton Company Inc., Reno, NV) was inserted through a burr hole into the right lateral ventricle using the following coordinates relative to the bregma: $0.9 \mathrm{~mm}$ posterior, $1.5 \mathrm{~mm}$ lateral, and $3.3 \mathrm{~mm}$ below the horizontal plane of the bregma. Drug and siRNA were infused at a rate of $1 \mu \mathrm{l} / \mathrm{min}$. The needle was removed 5 minutes after i.c.v infusion, and we quickly sealed the skull hole with bone wax, and then, the incision site was sutured. The dosage of siRNA was determined based on our previous in vivo report [5]. Negative control Scr siRNA and SPARC siRNA were prepared at a concentration of $100 \mathrm{pmol} / \mu \mathrm{l}$ in RNase free resuspension buffer. A total volume of $5 \mu \mathrm{l}$ siRNA was injected $48 \mathrm{~h}$ before SAH induction. The SPARC mAb 236 exerts function blocking effects by the action of the rounding (inhibition of spreading) activity of SPARC on endothelial cells [28]. The dosage of SPARC mAb 236 was determined based on a previous in vitro report [28]. In the study, $1 \mu \mathrm{g} / \mathrm{ml}$ SPARC mAb 236 significantly inhibited the adhesion of SPARC to endothelial cells. The dosage of SPARC mAb 236 was determined to achieve the equivalent working concentration of $1 \mu \mathrm{g} / \mathrm{ml}$ in cerebrospinal fluid (CSF) in rats, whose total CSF volume is presumed to be $300 \mu \mathrm{l}$ [29], and determined to be $0.3 \mu \mathrm{g}$. SPARC $\mathrm{mAb} 236$ and normal mouse IgG were prepared at a concentration of $41 \mu \mathrm{g} / \mathrm{ml}$ in PBS, and a total volume of $7.3 \mu \mathrm{l}$ of SPARC mAb 236 and normal mouse IgG were infused at $1 \mathrm{~h}$ after SAH induction. The dosage of rSPARC was determined based on a previous in vitro study [13]. In the study, $1 \mu \mathrm{g} / \mathrm{ml} \mathrm{rSPARC}$ significantly reduced the tight junction marker $\mathrm{ZO}-1$ and occludin in cultured human cerebral microvascular endothelial cells. To achieve an equivalent CSF concentration in rats, the dosage of rSPARC was determined to be $0.3 \mu \mathrm{g}$.

2.5. Intranasal Administration. Intranasal administration was carried out as previously described [5]. Briefly, rats were placed in a supine position under $2 \%$ isoflurane anesthesia. A total volume of $2.0 \mu \mathrm{g}$ cRGD was dissolved in $4 \mu \mathrm{l} \mathrm{PBS}$, and administered into the left nares $1 \mathrm{~h}$ before modeling. The cRGD has a molecular weight of 717.69 and a molecular formula of $\mathrm{C}_{27} \mathrm{H}_{41} \mathrm{~N}_{9} \mathrm{O}_{7}$, which can cross the BBB [30]. The dosage of $\mathrm{CRGD}$ was determined based on a previous in vitro report [31]. In this study, a concentration of $1.33 \mathrm{nmol} / \mathrm{l}$ cRGD showed $50 \%$ inhibition of signal $\left(\mathrm{IC}_{50}\right.$ value) for the inhibition of the vitronectin binding to integrin $\alpha \mathrm{V} \beta 3$. A 10-time higher concentration of $\mathrm{IC}_{50}$ value for $\mathrm{CRGD}$ was tentatively selected in this study. To achieve an equivalent concentration in rats, total body fluid volume is presumed to be $210 \mathrm{ml}$ (70\% of body weight) [32], and the dosage of cRGD was determined to be $2.0 \mu \mathrm{g}$.

2.6. Neurobehavioral Tests. Neurological impairments were blindly evaluated using the modified Garcia's neurological score and beam balance score system as previously described [5]. Briefly, the evaluation consisted of 6 categories (spontaneous activity, spontaneous movement of four limbs, forepaw outstretching, climbing, body proprioception, and response to whisker stimulation) that could be scored 0 to 3 or 1 to 3 . A neurological score was determined by adding the 6 scores to get a final score with 3 being the worst and 18, the best (Additional file 1: Figure S2a). The median score of 3 consecutive trials in a $5 \mathrm{~min}$ interval was calculated. A beam balance test investigated animal's ability to walk on a narrow wooden beam for 60 seconds, and we blindly marked a grade from 0 to 4 points as follows: 4 points, walking $\geq 20 \mathrm{~cm} ; 3$ points, walking $<20 \mathrm{~cm} ; 2$ points, walking but falling; 1 point, not walking while remaining on beam; and 0 points, falling without walking (Additional file 1: Figure S2b). The median score of 3 consecutive trials in a $5 \mathrm{~min}$ period was calculated. 
2.7. Brain Water Content. Brain edema was determined using the wet/dry method as previously described [5]. Briefly, after sacrificing rats under deep anesthesia, the brain was quickly removed, separated into 4 segments (left and right cerebral hemispheres, cerebellum, and brain stem), and weighed immediately as wet weight. The brain specimens were dried in an oven at $105^{\circ} \mathrm{C}$ for $72 \mathrm{~h}$ and weighed again as dry weight. The water content of each specimen was calculated according to the following formula: [(wet weight - dry weight)/wet weight] $\times 100 \%$.

2.8. Immunofluorescence Staining. An immunofluorescence staining was performed as previously described [5]. Under deep anesthesia, rats were sacrificed by transcardial perfusion with $100 \mathrm{ml}$ PBS followed by 2 minutes of $10 \%$ neutral buffered formalin. Brains were fixed in 10\% neutral buffered formalin for $24 \mathrm{~h}$ at $4^{\circ} \mathrm{C}$ followed by $30 \%$ sucrose solution for another $72 \mathrm{~h}$. After being frozen at $-80^{\circ} \mathrm{C}$, the brain was cut into $10 \mu \mathrm{m}$ thick coronal sections at $1.0 \mathrm{~mm}$ posterior to the bregma using a cryostat (LM3050S; Leica Microsystems, Bannockburn, III, Germany). Slides were washed with $0.01 \mathrm{M}$ of PBS three times for 10 minutes then incubated in $0.3 \%$ Triton $\mathrm{X}-100$ in $0.01 \mathrm{M}$ of PBS for 30 minutes at room temperature. After being blocked with $10 \%$ donkey serum in $0.01 \mathrm{M}$ of PBS for $1 \mathrm{~h}$ at room temperature, the sections were incubated at $4^{\circ} \mathrm{C}$ overnight with the primary antibody as follows: goat antiglial fibrillary acidic protein (GFAP; 1:500; ab104224, Abcam Inc., Cambridge, MA), mouse anti-von Willebrand factor (vWF; 1:400; sc-365712, Santa Cruz Biotechnology Inc., Dallas, TX), rabbit anti-SPARC (1:500; NBP1-80972, Novus Biologicals Inc., Centennial, CO), rabbit antiintegrin $\alpha \mathrm{V} \beta 3$ (1:50; SC56-07, Novus Biologicals Inc., Centennial, CO), rabbit antiphosphorylated c-Jun Nterminal kinase (p-JNK; 1:100; ab131499, Abcam Inc., Cambridge, MA), and rabbit antiphosphorylated p38 (pp38; $1: 400$; 9211, Cell Signaling Technology Inc., Danvers, MA). Then, the sections were washed with $0.01 \mathrm{M}$ of PBS and incubated with the appropriate fluorescenceconjugated secondary antibodies (1:200; Jackson ImmunoResearch Inc., West Grove, PA) for $1 \mathrm{~h}$ at room temperature. The slides were observed and photographed under a fluorescence microscope (DMi8; Leica Microsystems Inc., Germany; $\times 20$ ). The densitometric analysis of $\mathrm{p}-\mathrm{JNK}$ and p-p38 was performed using Image Pro Plus 6.0 software (Media Cybernetics Inc., Rockville, MD) in the 4 continuous pictures of the left (perforation side) temporal cortex based on our previous study [4].

2.9. Immunohistochemical Staining of Immunoglobulin $G$ (IgG). Immunohistochemical staining of IgG was performed to evaluate $\mathrm{BBB}$ permeability using a commercially available kit (Vectastain ABC Peroxidase kit; PK-4004, Vector Laboratories, Burlingame, CA). Coronal brain sections were prepared in the same way as immunofluorescence staining. After being washed with $0.01 \mathrm{M}$ of PBS three times for 10 minutes, slides were incubated in $0.3 \%$ Triton X-100 in $0.01 \mathrm{M}$ of PBS for 30 minutes at room temperature. After being incubated in $3 \%$ hydrogen perox- ide $\left(\mathrm{H}_{2} \mathrm{O}_{2}\right)$ for 20 minutes to quench any endogenous peroxidase activity, the sections were blocked with $10 \%$ rabbit normal serum in $0.01 \mathrm{M}$ of PBS for $1 \mathrm{~h}$ at room temperature followed by overnight incubation at $4^{\circ} \mathrm{C}$ with biotinylated rabbit anti-rat polyclonal IgG and then $1 \mathrm{~h}$ incubation at room temperature with an avidin-biotin-horseradish peroxidase complex. Color reactions were developed in diaminobenzidine $/ \mathrm{H}_{2} \mathrm{O}_{2}$ solution, and the sections were lightly counterstained with hematoxylin. To evaluate the amount of IgG extravasation, 4 continuous pictures of the left temporal cortex were photographed under light microscope $(\times 20)$, and the relative quantity of extravasated IgG was calculated by Image Pro Plus 6.0 software (Media Cybernetics Inc., Rockville, MD). The left (perforation side) temporal cortex was selected based on our previous study [4].

2.10. Western Blot. The left cerebral hemisphere was separated and utilized. Equal amounts of protein samples $(30 \mu \mathrm{g})$ were loaded on SDS-PAGE gels, electrophoresed, and transferred onto a polyvinylidene difluoride membrane. The membranes were blocked with $5 \%$ bovine serum albumin in $0.01 \mathrm{M}$ of PBS for $2 \mathrm{~h}$ at room temperature followed by incubation overnight at $4^{\circ} \mathrm{C}$ with the following antibodies: rabbit anti-SPARC $(1: 2000$; NBP1-80972, Novus Biologicals Inc., Centennial, CO), rabbit anti-integrin $\alpha \mathrm{V} \beta 3$ (1:1000; SC56-07, Novus Biologicals Inc., Centennial, CO), mouse anti- $\beta$-actin ( $1: 5000 ;$ sc-47778, Santa Cruz Biotechnology Inc., Dallas, TX), rabbit anti-p-JNK (1:1000; ab131499, Abcam Inc., Cambridge, UK), mouse anti-JNK (1:5000; 66210-1-Ig, Proteintech Group Inc., Rosemont, IL), rabbit anti-p-p38 (1:1000; 9211, Cell Signaling Technology Inc., Danvers, MA), rabbit anti-p38 (1:1000; 9212, Cell Signaling Technology Inc., Danvers, MA), rabbit anti-IL-6 (1:1000; PR627, Thermo Fisher Scientific Inc., Waltham, MA), rabbit anti-MMP-9 (1:1000; ab182734, Abcam Inc., Cambridge, UK), rabbit anti-ZO-1 ( $1: 1000 ; 61-7300$, Thermo Fisher Scientific Inc., Waltham, MA), and rabbit anti-VE-cadherin antibody (1:1000; ab33168, Abcam Inc., Cambridge, UK). On the following day, the membranes were incubated with the appropriate secondary antibody (1:5000; sc-516102, Santa Cruz Biotechnology Inc., Dallas, TX, and 1:5000; 12-348, MilliPore sigma Inc., Temecura, CA) at room temperature for $2 \mathrm{~h}$. Immunoreactive bands were detected with a chemiluminescence reagent kit (ECL Prime; Amersham Biosciences Inc., Arlington Heights, IL) and quantified by densitometry with Image J software (NIH, Bethesda, MD).

2.11. Statistical Analysis. Neurological score and SAH grade were expressed as median \pm 25 th -75 th percentiles and were compared with Kruskal-Wallis tests followed by Steel-Dwass multiple comparisons. Brain water content, immunohistochemical staining of $\mathrm{IgG}$, and $\mathrm{WB}$ results were expressed as mean \pm standard deviation and were compared with one-way analysis of variance (ANOVA) followed by Tukey-Kramer post hoc tests. Statistical analyses were performed using SPSS version 23.0 (IBM, Tokyo, Japan). A value of $P<0.05$ was considered significant. 


\section{Results}

3.1. General Observation. Comparisons of physiological parameters revealed no significant differences among the groups (data not shown). A total of 197 rats underwent endovascular perforation to induce SAH or sham operation. The mortality was 0 of $54(0 \%)$ in the sham-operated rats, and it was 16 of $147(11 \%)$ in the SAH-operated rats in total during the observation period in all experiments. A total of 15 rats were not used for the analyses because of mild $(\leq 7)$ SAH grade (Additional file 1: Figure S3a). There was no significant difference in SAH grade among all $\mathrm{SAH}$ groups at $24 \mathrm{~h}$ after SAH (Additional file 1: Figure S3b, c). Brain water content, immunostaining of $\mathrm{IgG}$, and $\mathrm{WB}$ results were normally distributed and had homogeneity of variance.

3.2. Time Course and Spatial Expression of SPARC and Integrin $\alpha V \beta 3$ after $S A H$. WB results showed that expression of SPARC peaked at $12 \mathrm{~h}$ after SAH induction, and the elevation was prolonged at least until $72 \mathrm{~h}$ when compared to the sham group (Figure 1(a)). In contrast, the expression of integrin $\alpha \mathrm{V} \beta 3$ was significantly elevated from $6 \mathrm{~h}$ to at least $72 \mathrm{~h}$ with the peak at $24 \mathrm{~h}$ from SAH induction. Double immunofluorescence staining showed that SPARC was expressed in astrocytes and endothelial cells in the cerebral cortex in both sham and SAH groups at $24 \mathrm{~h}$ from modeling, and integrin $\alpha \mathrm{V} \beta 3$ was expressed in endothelial cells at $24 \mathrm{~h}$ post-SAH (Figure 1(b)).

3.3. Knockdown of SPARC Suppressed Neurological Impairments, Brain Edema, and BBB Permeability after $S A H$. The silencing efficacy of SPARC siRNA was validated by WB and immunofluorescence staining in the left cerebral hemisphere at $72 \mathrm{~h}$ after siRNA injection (Additional file 1: Figure S4a, b). After SPARC siRNA was injected, post$\mathrm{SAH}$ aggravation of neurological scores significantly decreased in both modified Garcia's and beam balance tests, while Scr siRNA did not have an effect after SAH (Figure 2(a)). In addition, brain water content in the left cerebral hemisphere $24 \mathrm{~h}$ post-SAH significantly improved in the SAH + SPARC siRNA, while Scr siRNA administration had no significant effects on brain water content after SAH (Figure 2(b)). Immunohistochemical staining of IgG was performed to evaluate the severity of $\mathrm{BBB}$ disruption in the left cerebral cortex $24 \mathrm{~h}$ after SAH (Figures 3(a)-3(d)). SAH + Scr siRNA group resulted in a significant increase in IgG extravasation $24 \mathrm{~h}$ post-SAH, which was significantly suppressed in SAH + SPARC siRNA group (Figure 3(e)). At $72 \mathrm{~h}$, SPARC siRNA also inhibited post-SAH aggravation of neurological score in the modified Garcia's test and brain edema in the left cerebral hemisphere and cerebellum compared with Scr siRNA (Figure 4).

3.4. Endogenous SPARC May Aggravate Post-SAH Neurological Deficits and BBB Disruption via MAPKs p$J N K$ and $p$-p38. A SPARC mAb 236 improved neurological scores and brain edema in the left hemisphere compared with negative control normal mouse IgG after SAH (Figures 5(a) and 5(b)). Immunohistochemical staining of
IgG showed that SPARC mAb 236 administration resulted in a significant suppression of IgG extravasation $24 \mathrm{~h}$ postSAH compared with normal mouse IgG (Figures 6(a)6(d)). Expression of two major MAPKs p-JNK and p-p38 in the endothelial cells was assessed by double immunofluorescence staining. SAH rats treated with normal mouse IgG showed the upregulation of p-JNK and p-p38 while SPARC $\mathrm{mAb} 236$ treatment inhibited the expression of $\mathrm{p}-\mathrm{JNK}$ and p-p38 in endothelial cells. The densitometry of p-JNKpositive cells and p-p38-positive cells were significantly higher after SAH induction, which were significantly suppressed by SPARC mAb 236 administration (Figures 6(e) and $6(\mathrm{f}))$.

3.5. Integrin $\alpha V \beta 3 / M A P K$ Signaling Pathway May Make a Greater Contribution to SPARC-Mediated BBB Disruption after $S A H$. A rSPARC did not affect neurological scores in sham-operated rats. However, rSPARC treatment significantly aggravated the neurological scores in post-SAH rats compared with PBS. Selective integrin $\alpha \mathrm{V} \beta 3$ inhibitor cRGD significantly reduced post-SAH harmful neurological effects of rSPARC (Figure 7(a)). WB analyses also demonstrated that $\mathrm{rSPARC}$ did have an effect on the expression of integrin $\alpha \mathrm{V} \beta 3$ and the intracellular downstream mediators in shamoperated rats. In contrast, post-SAH rSPARC administration enhanced the expression of integrin $\alpha \mathrm{V} \beta 3$, p-p38, and active MMP-9 and reduced the expression of tight junction marker ZO-1 compared with PBS. The protective effects of cRGD were associated with a significant suppression of post-SAH activation of MAPKs including p-p38 and p-JNK, proinflammatory mediator IL-6, and MMP-9 followed by retention of endothelial tight junction protein $\mathrm{ZO}-1$ and adherens junction protein VE-cadherin (Figure 7(b)).

\section{Discussion}

The novel findings in the present study are as follows: (1) the expression of SPARC and integrin $\alpha \mathrm{V} \beta 3$ is upregulated in endovascular perforation SAH rats; (2) knockdown of SPARC and functional blocking against endogenous SPARC prevented neurological impairments through protection of the BBB as measured by IgG extravasation after SAH; (3) exogenous SPARC aggravated neurobehavioral impairments after $\mathrm{SAH}$, and integrin $\alpha \mathrm{V} \beta 3$ inhibitor suppressed the harmful neurological effects of SPARC via inhibition of two MAPKs JNK and p38 and MMP-9 followed by retention of ZO-1 and VE-cadherin.

The post-SAH time course of SPARC and integrin $\alpha \mathrm{V} \beta 3$ levels have never been determined, while expression of integrin $\alpha \mathrm{V} \beta 3$ in microvessels after experimental ischemic stroke is significantly increased at $2 \mathrm{~h}$ poststroke in adult baboons [33]. The present study demonstrated that the expression of both SPARC and integrin $\alpha \mathrm{V} \beta 3$ was upregulated within $12 \mathrm{~h}$, and, therefore, SPARC and integrin $\alpha \mathrm{V} \beta 3$ signaling pathways are possibly involved in acute phase brain injury after SAH. An in vitro study demonstrated that proinflammatory cytokine tumor necrosis factor- (TNF-) $\alpha$ increased SPARC expression in cerebral endothelium [13]. In contrast, SPARC knockdown reduced 


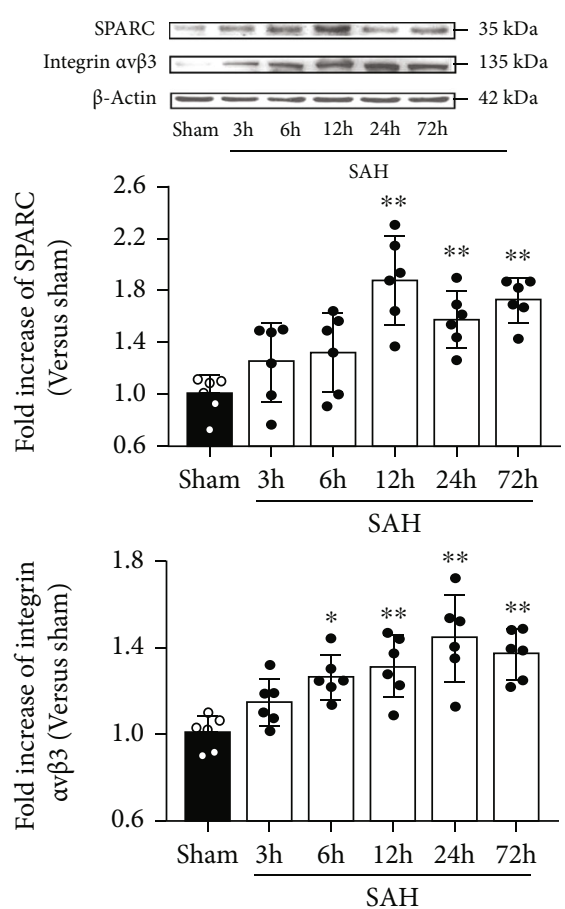

(a)
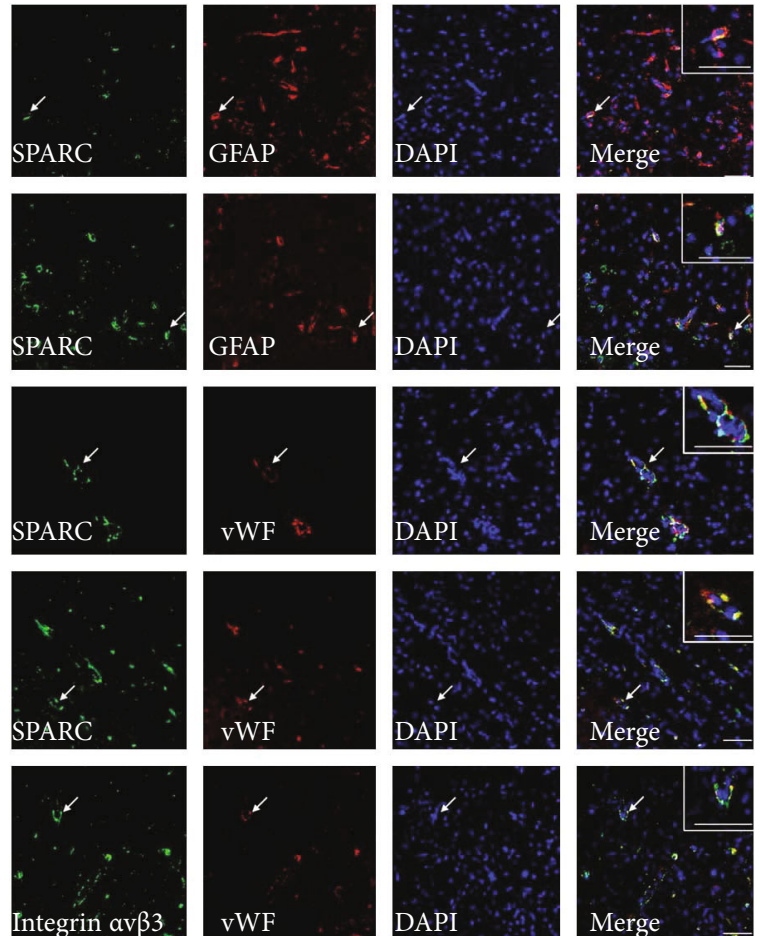

(b)

FIGURE 1: Expression of endogenous secreted protein acidic and rich in cysteine (SPARC) and integrin $\alpha \mathrm{V} \beta 3$. (a) Representative Western blot images and densitometric quantification of time-dependent expression of SPARC and integrin $\alpha \mathrm{V} \beta 3$ after subarachnoid hemorrhage (SAH). Expression levels of each protein are divided by the levels of $\beta$-actin and expressed as a ratio of the average levels of sham models for normalization (mean \pm standard deviation; $n=6$ per group). ${ }^{*} P<0.05$ and ${ }^{* *} P<0.01$ vs. sham group. (b) Colocalization of SPARC (green) and integrin $\alpha \mathrm{V} \beta 3$ (green) with glial fibrillary acidic protein (GFAP; red) or von Willebrand factor (vWF; red) in the left temporal cerebral cortex at $24 \mathrm{~h}$ after modeling. Nuclei are stained with DAPI (blue). Inset highlighting higher magnification image of SPARC and integrin $\alpha \mathrm{V} \beta 3$ colocalized with GFAP or vWF (indicated as arrows). Scale bar $=50 \mu \mathrm{m}$ ( $n=2$ per group).
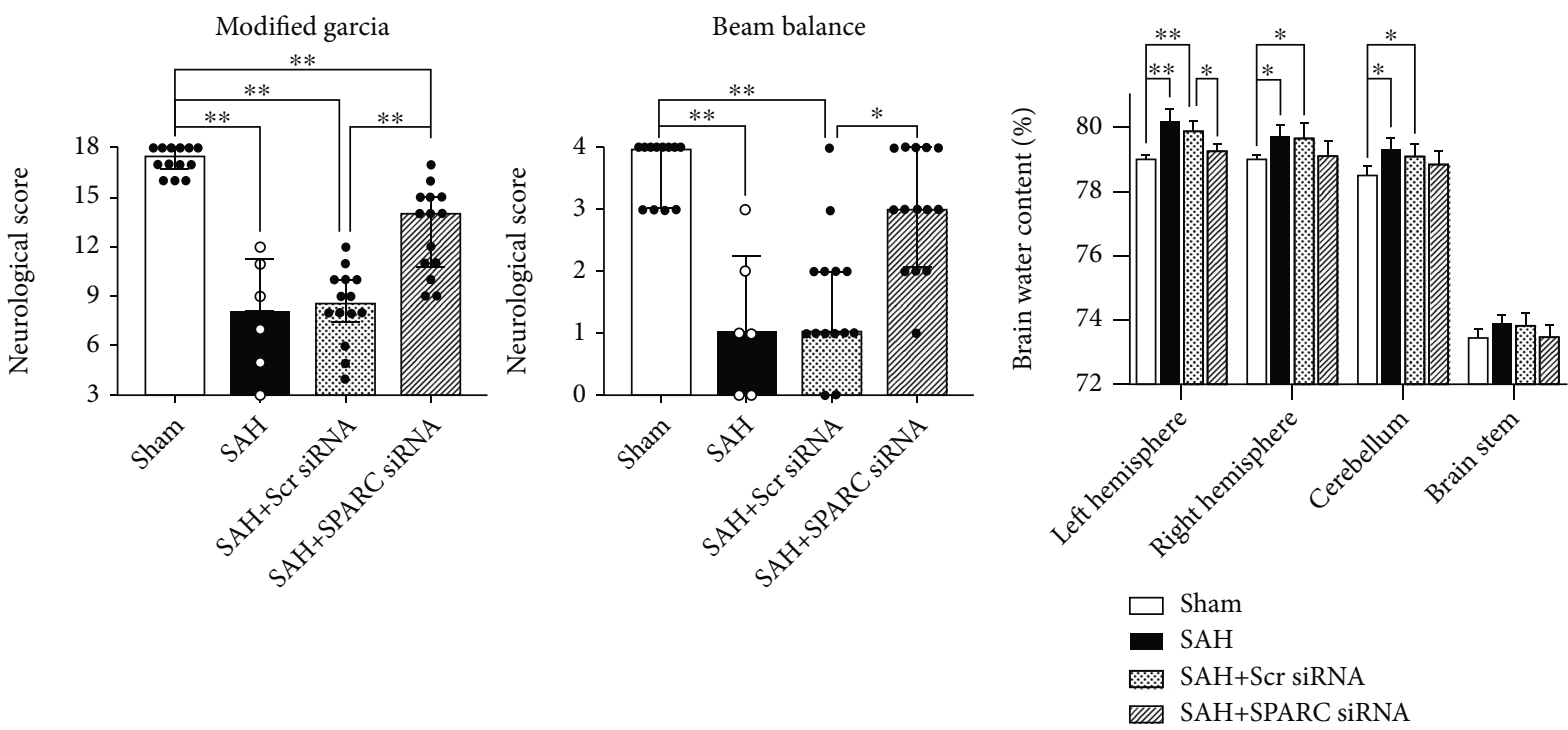

(a)

(b)

FIGURE 2: Effects of knockdown of a secreted protein acidic and rich in cysteine (SPARC) on neurobehavioral function and brain edema at $24 \mathrm{~h}$ after modeling. (a) SPARC small interfering ribonucleic acid (siRNA) improves modified Garcia's score and beam balance score after subarachnoid hemorrhage (SAH). Data are expressed as median \pm 25 th-75th percentiles $\left(n=6\right.$-14 per group). ${ }^{*} P<0.05$ and ${ }^{* *} P<0.01$. (b) SPARC siRNA treatment improves brain water content after SAH. Data are expressed as mean \pm standard deviation $\left(n=6\right.$ per group). ${ }^{*} P$ $<0.05$ and ${ }^{* *} P<0.01$. Scr siRNA: scrambled siRNA. 


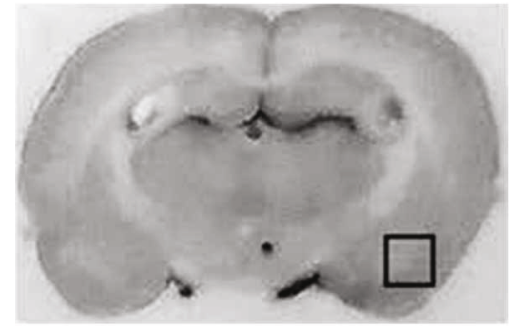

(a)

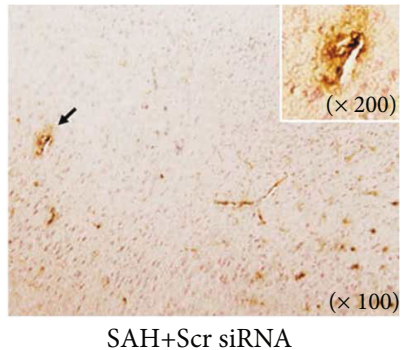

(c)

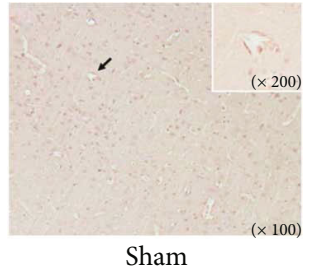

(b)

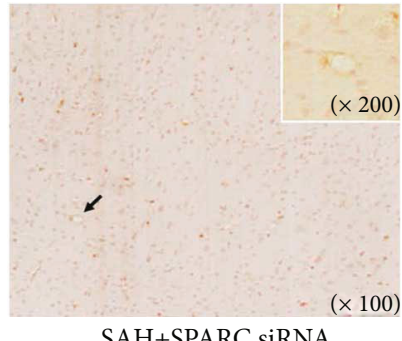

(d)

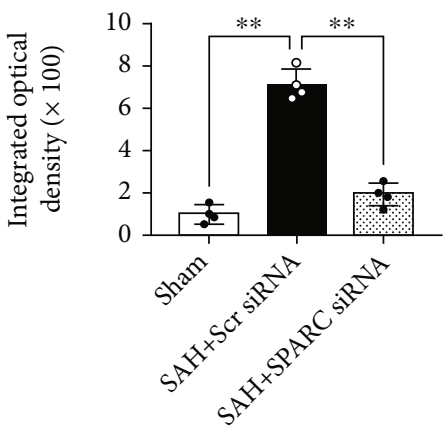

(e)

FIGURE 3: Effects of a secreted protein acidic and rich in cysteine (SPARC) small interfering ribonucleic acid (siRNA) on blood-brain barrier permeability at $24 \mathrm{~h}$ after modeling. (a) Representative brain slice showing the location of staining (small box) in the left temporal cortex at $1.0 \mathrm{~mm}$ posterior to the bregma. (b-d) Representative immunohistochemical staining of immunoglobulin $\mathrm{G}$ (IgG) in sham-operated rats (b), subarachnoid hemorrhage (SAH) rats treated with scrambled siRNA (Scr siRNA) (c), and SAH rats treated with SPARC siRNA (d). Inset highlighting higher magnification image of endothelial cells (indicated as arrows). (e) The sum of integrated optical density of IgG. Data are expressed as mean \pm standard deviation $\left(n=4\right.$ per group). ${ }^{* *} P<0.01$.

expression of TNF- $\alpha$ and IL- 6 and protected vascular endothelial cells from damage [34]. In addition, SPARC was regulated by MMPs [35], while SPARC has been shown to induce the production and activity of MMPs [36-38]. SPARC interacting with TNF- $\alpha$ and MMPs may amplify the expression levels of each other in an acute phase.

An in vitro study demonstrated that SPARC increased transendothelial albumin flux in a dose-dependent manner at concentrations. At a fixed dose, longer exposure times induced albumin flux, which was blocked by anti-SPARC antibodies [39]. Goldblum et al. [39] demonstrated that SPARC promoted barrier dysfunction in endothelial cell monolayers. Tilling et al. [40] demonstrated that SPARC inhibited collagen IV-mediated increase in transendothelial electrical resistance (TEER) of low TEER brain capillary endothelial cells. TEER value is a widely accepted technique to measure the integrity of tight junction including $\mathrm{BBB}$ [41]. The findings of these studies indicated that SPARC increased the permeability of endothelium. However, the effects of SPARC in hemorrhagic stroke have been poorly understood. The present study demonstrated that endogenous SPARC was induced in reactive astrocytes and capillary endothelial cells in an acute phase of SAH and that immediate treatment to block endogenous SPARC exerted BBB protection effects in SAH while exogenous SPARC exerted further harmful neurological effects. These results suggest that SPARC aggravates post-SAH BBB disruption in an acute phase.

BBB is mainly composed of a chemical and structural barrier formed by endothelial junction-associated proteins, such as ZO-1 and VE-cadherin, a basal lamina, a pericyte, and an astrocytic perivascular end-feet [18, 42, 43]. Degradation of the junction-associated proteins opens endothelial cell-cell junction and increases BBB permeability [18, 44]. A proteolytic enzyme MMP-9 has been repeatedly reported to cause post-SAH BBB disruption by degrading the cerebral 

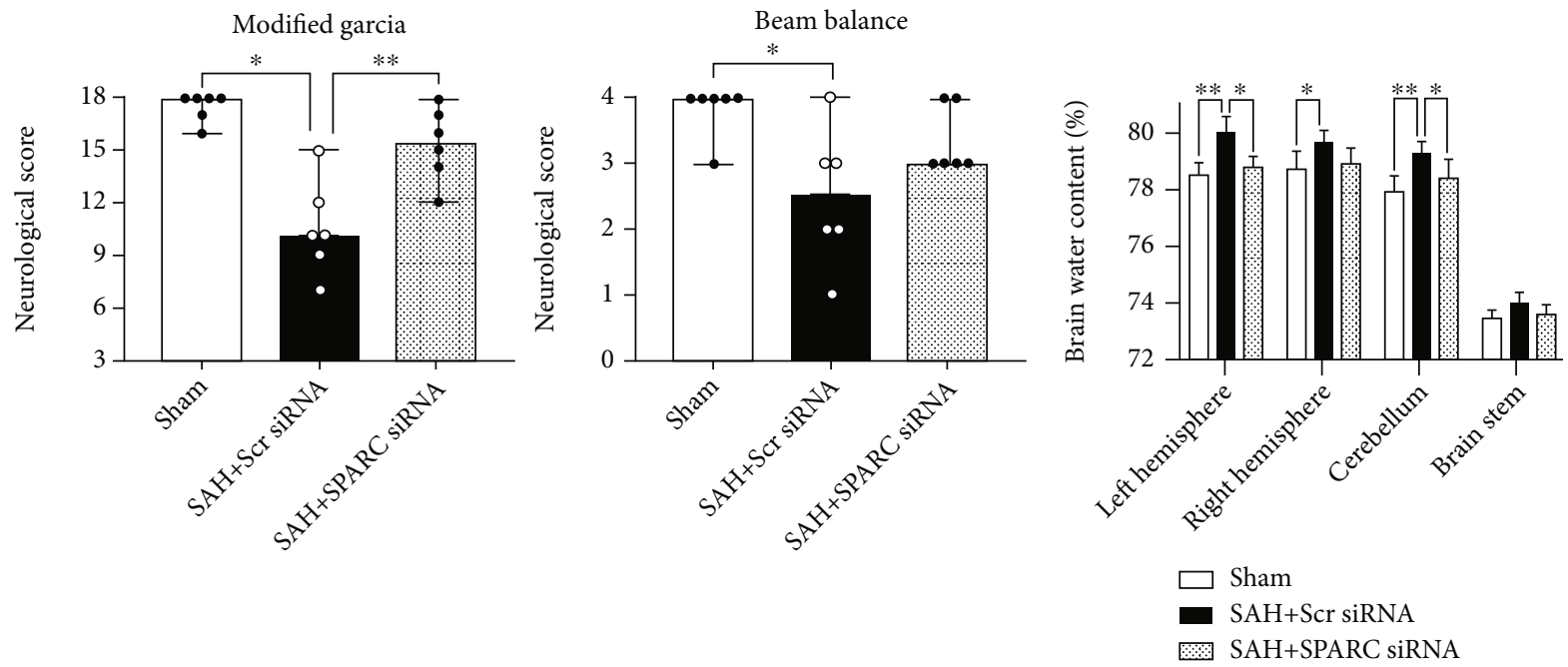

(a)

(b)

FIGURE 4: Effects of knockdown of a secreted protein acidic and rich in cysteine (SPARC) on neurobehavioral function and brain edema at $72 \mathrm{~h}$ after modeling. (a) SPARC small interfering ribonucleic acid (siRNA) improves modified Garcia's score after subarachnoid hemorrhage (SAH). Data are expressed as median \pm 25 th-75th percentiles $\left(n=6\right.$ per group). ${ }^{*} P<0.05$ and ${ }^{* *} P<0.01$. (b) SPARC siRNA treatment improves brain water content after SAH. Data are expressed as mean \pm standard deviation ( $n=6$ per group). ${ }^{*} P<0.05$ and ${ }^{* *} P<0.01$. Scr siRNA: scrambled siRNA.

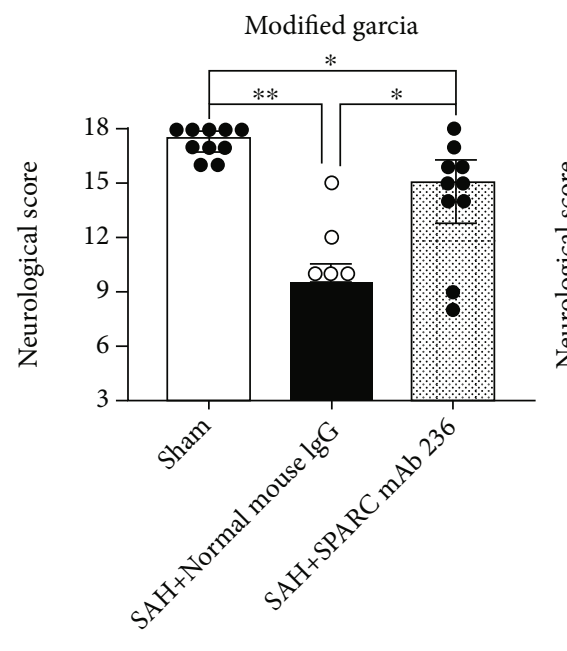

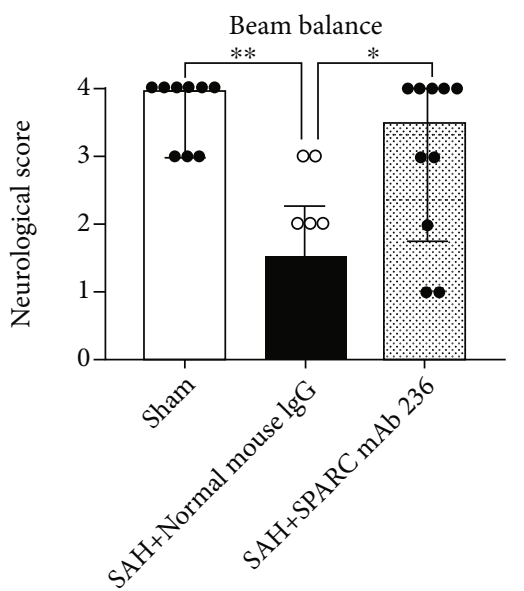

(a)

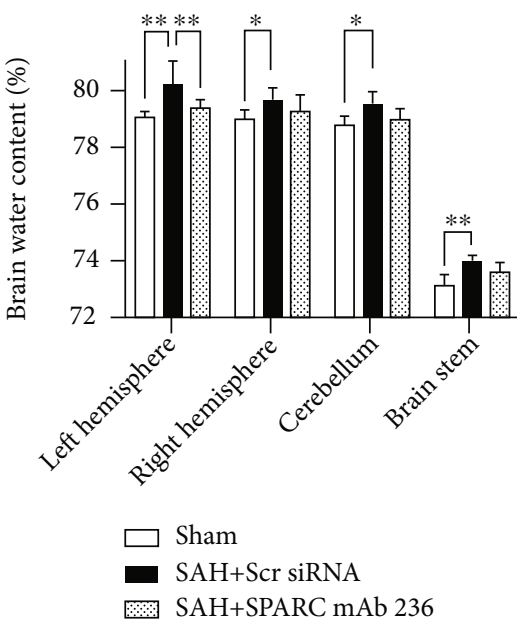

(b)

FIGURE 5: Effects of a functional blocking against secreted protein acidic and rich in cysteine (SPARC) on neurobehavioral function and brain edema at $24 \mathrm{~h}$ after modeling. (a) SPARC monoclonal antibody (mAb) 236 improves modified Garcia's score and beam balance score after subarachnoid hemorrhage (SAH). Data are expressed as median \pm 25 th-75th percentiles $\left(n=10\right.$ per group). ${ }^{*} P<0.05$ and ${ }^{* *} P$ $<0.01$. (b) SPARC mAb 236 treatment improves brain water content after SAH. Data are expressed as mean \pm standard deviation ( $n=10$ per group). ${ }^{*} P<0.05$ and ${ }^{* *} P<0.01$. IgG: immunoglobulin $\mathrm{G}$.

microvessel basal lamina and endothelial tight junction protein $\mathrm{ZO}-1$ and adherens junction protein VE-cadherin [45-50]. Among the MMP family, MMP-2 also exerts proteolytic effects of endothelial cells only within several hours after experimental ischemic stroke, although other studies have shown no obvious increases in MMP-2 expression [45]. However, MMP-2 activity did not change in brain after filament perforation SAH, while MMP-9 activity was upreg- ulated in our previous study using zymography [51]. Therefore, this study focused on the involvement of SPARCinduced MMP-9 induction after SAH. MCPs other than SPARC also have either beneficial or detrimental effects on BBB via MMP-9 in SAH [18, 24, 42, 46, 49, 52]. Our recent studies demonstrated that three kinds of MCPs, tenascin-C, periostin, and galectin-3, were induced and caused BBB disruption after SAH via MAPK-mediated activation of MMP- 


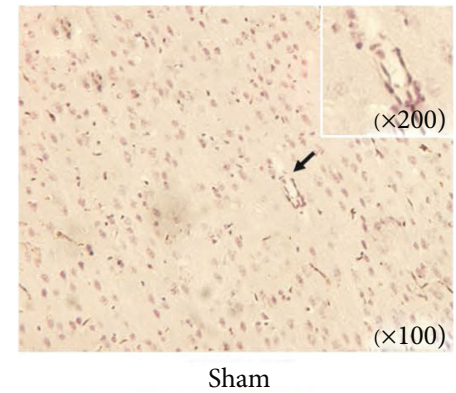

(a)

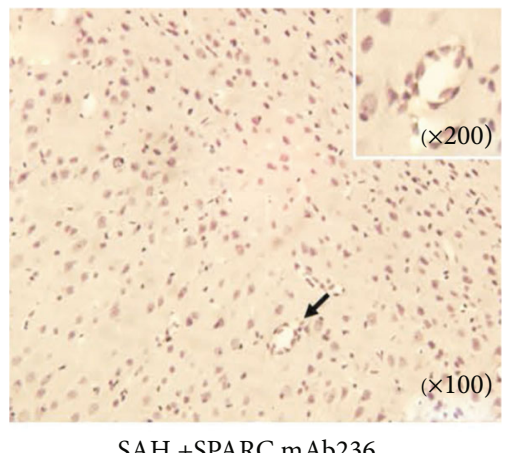

SAH +SPARC mAb236

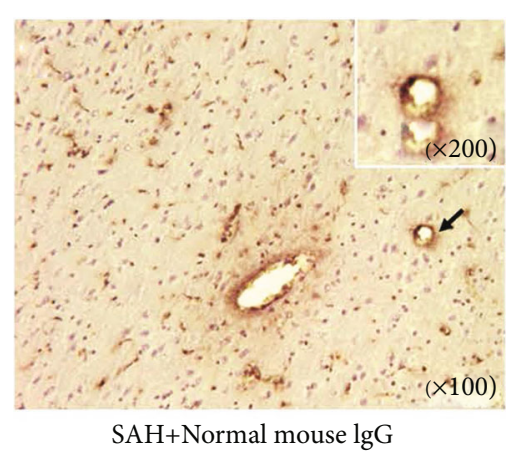

(b)

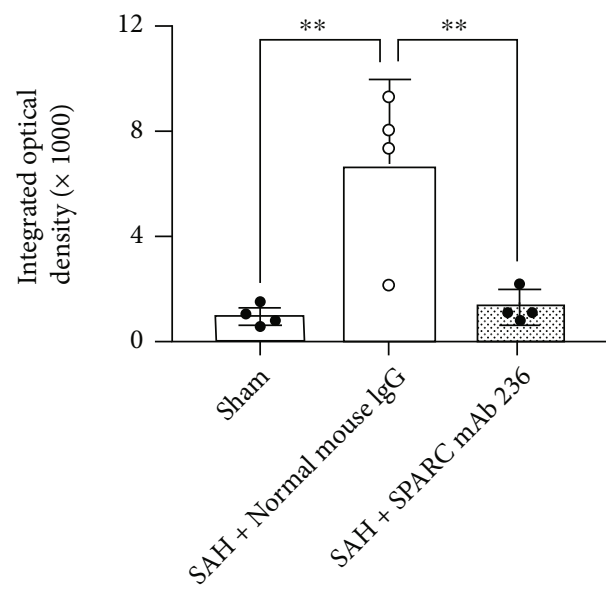

(d)
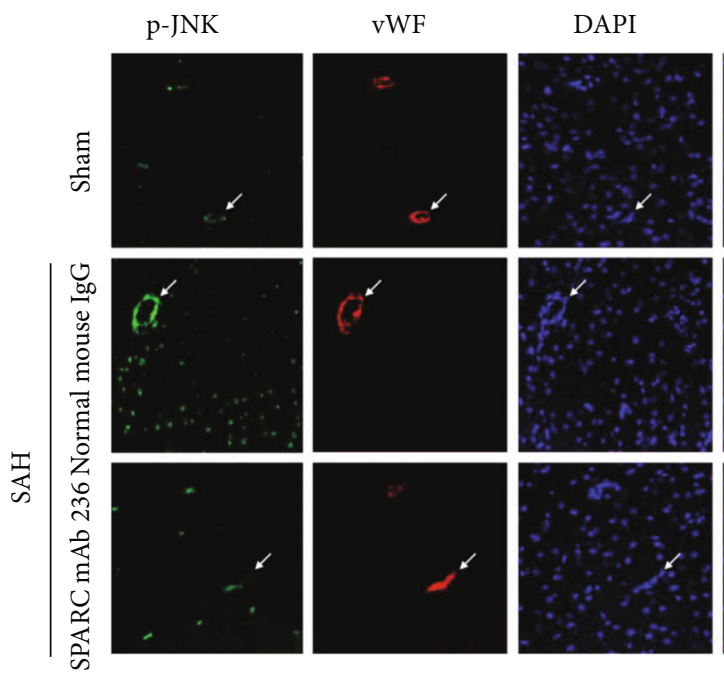

Merge
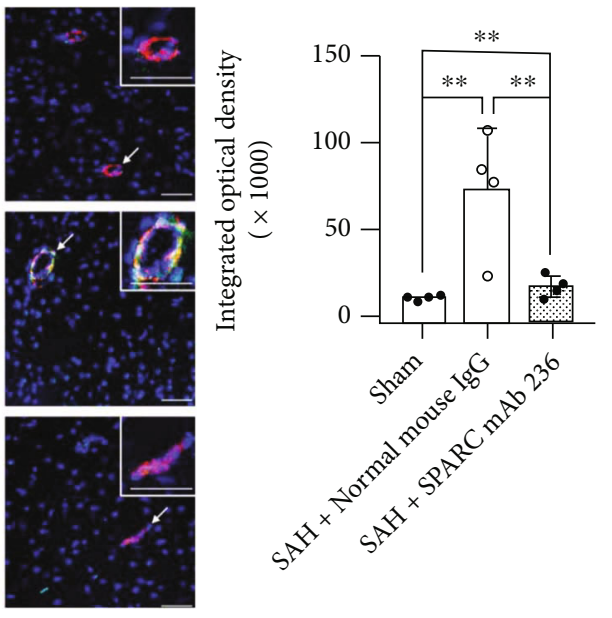

(e)

Figure 6: Continued. 

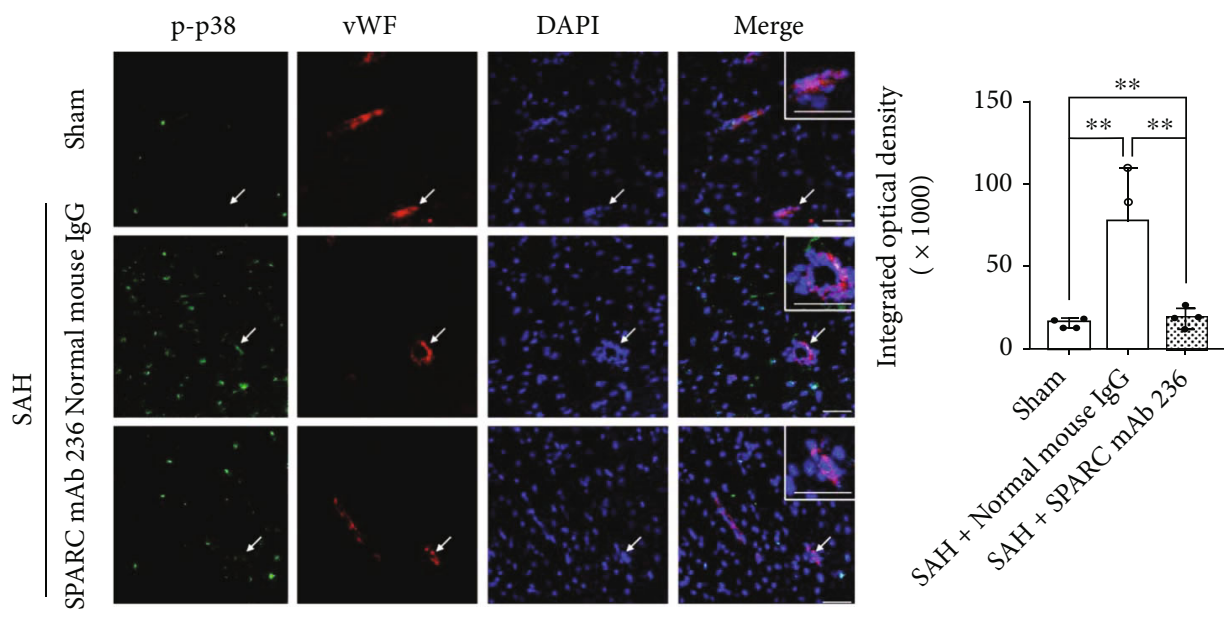

(f)

FIGURE 6: Effects of a functional blocking against secreted protein acidic and rich in cysteine (SPARC) on blood-brain barrier permeability and expression of phosphorylated c-Jun N-terminal kinase (p-JNK) and phosphorylated p38 (p-p38) in endothelial cells at 24h after modeling. (a-c) Representative immunohistochemical staining of immunoglobulin G (IgG) in the left temporal cortex at $1.0 \mathrm{~mm}$ posterior to the bregma in sham-operated rats (a), subarachnoid hemorrhage (SAH) rats treated with normal mouse IgG (b), and SAH rats treated with SPARC monoclonal antibody (mAb) 236 (c). Inset highlighting higher magnification image of endothelial cells (indicated as arrows). (d) The sum of integrated optical density of IgG. Data are expressed as mean \pm standard deviation ( $n=4$ per group). ${ }^{* *} P<0.01$. (e, f) Representative immunohistochemical staining of p-JNK (green (e)) and p-p38 (green (f)) in the left temporal cortex at $1.0 \mathrm{~mm}$ posterior to the bregma and densitometric analyses of p-JNK-positive cells (e) and p-p38-positive cells (f). Nuclei are stained with DAPI (blue). Inset highlighting higher magnification image of von Willebrand factor (vWF) positive cells (indicated as arrows). Scale bar $=50 \mu \mathrm{m}$. Data are expressed as mean \pm standard deviation $\left(n=4\right.$ per group). ${ }^{*} P<0.05$ and ${ }^{* *} P<0.01$.

9 and subsequent ZO-1 degradation in brain capillary endothelial cells in mice $[18,42,46,52]$. In contrast, another MCP osteopontin was induced in reactive astrocytes and capillary endothelial cells and prevented MMP-9 activation and BBB disruption by inactivating MAPK and nuclear factor- $\kappa \mathrm{B}[49,53]$. MAPK activation upregulates proinflammatory cytokines such as TNF- $\alpha$, IL-1 $\beta$, and IL-6, and MMP-9 in SAH $[45,54,55]$, and a MAPK inhibitor has been reported to protect the BBB integrity by MMP-9 downregulation and inactivation [56]. The present study showed that exogenous SPARC reduced the expression of tight junction protein $\mathrm{ZO}-1$ via more activation of $\mathrm{p} 38 \mathrm{MAPK}$ and then MMP-9 after SAH. In contrast, integrin $\alpha \mathrm{V} \beta 3$ inhibitor suppressed the harmful neurological effects of exogenous SPARC via inhibition of two forms of MAPKs JNK and p38 and then MMP-9 followed by retention of ZO-1 and VE-cadherin. Therefore, BBB disruption caused by SPARC induction after SAH possibly depends on integrin $\alpha \mathrm{V} \beta 3 /$ MAPK signaling pathway.

There are several limitations in this study that need to be noted. First, the SPARC signaling pathway that would induce post-SAH BBB disruption was not completely teased apart, due in part to the fact that other receptors, MCPs, growth factors, and cytokines have a high affinity with SPARC [21]. Moreover, other downstream mediators besides MAPKs were not examined in this study. A study demonstrated that SPARC has counteradhesive properties that result in rounding of endothelial cells, inhibition of endothelial cell spreading, and loss of focal adhesions through a tyrosine-kinase-dependent pathway [57]. Therefore, other protein-binding SPARC and the downstream sig- naling pathway influencing post-SAH BBB disruption should be examined in the future. Second, we cannot exclude the possibility that SPARC siRNA and SPARC mAb 236 inactivate other neuroreceptors and neuroregulatory molecules known to be involved in BBB disruption and neurological impairments rather than SPARC. Third, only i.c.v treatment with SPARC siRNA before SAH and SPARC $\mathrm{mAb} 236$ at $1 \mathrm{~h}$ after SAH was tested for the effects of SPARC inactivation in this study. As cerebrospinal fluid drainage is often placed in severe SAH patients to promote $\mathrm{SAH}$ clearance or to control acute hydrocephalus, an i.c.v. injection of this study could be applied to clinical settings. In order to be more translational, however, the effects of multiple treatments at different dosages or time courses and more simple delivery routes such as intravenous injections should be tested before future clinical trials. Fourth, endogenous ligands that activated integrin $\alpha \mathrm{V} \beta 3$ other than SPARC were not investigated in this study. For instance, vascular endothelial growth factor, a known inducer of $\alpha \mathrm{V} \beta 3$ integrin expression, also is upregulated after $\mathrm{SAH}$ and causes post-SAH BBB disruption [21, 25, 58]. Further studies are required to determine whether other factors released following SAH stimuli enhance activation of integrin $\alpha \mathrm{V} \beta 3$. Fifth, we evaluated SAH severity using grading score in addition to neurobehavioral tests. Our previous studies have repeatedly shown that the severity of brain injury was linearly correlated with SAH grading score at least during an acute phase of SAH $[5,59]$. However, it would be useful to demonstrate more experimental evidences for successful SAH modeling with uniform brain injuries. In addition, we did not monitor the tissue distribution of SPARC siRNA and $\mathrm{mAb}$ and their 


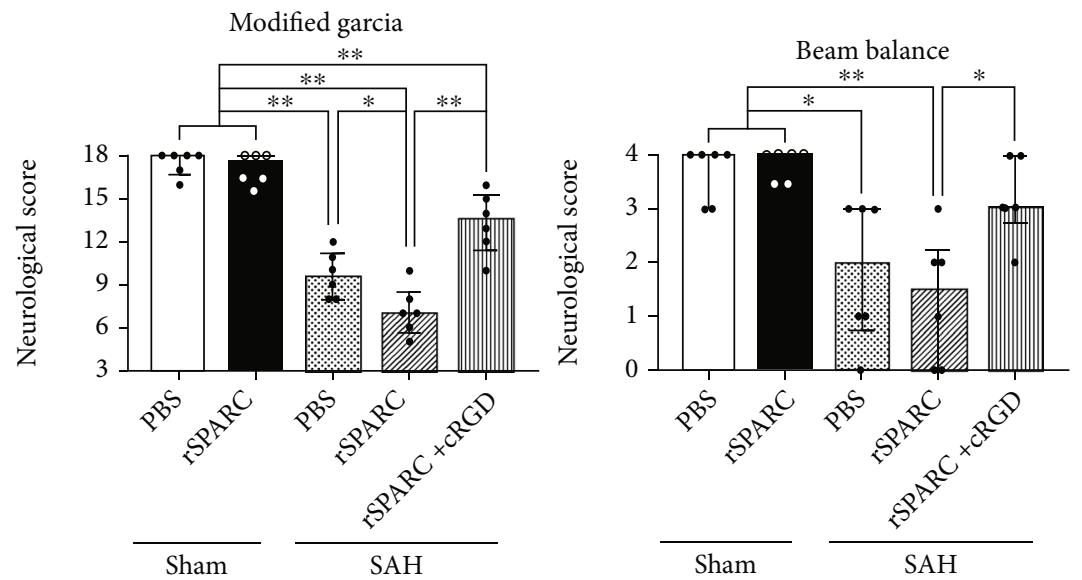

(a)
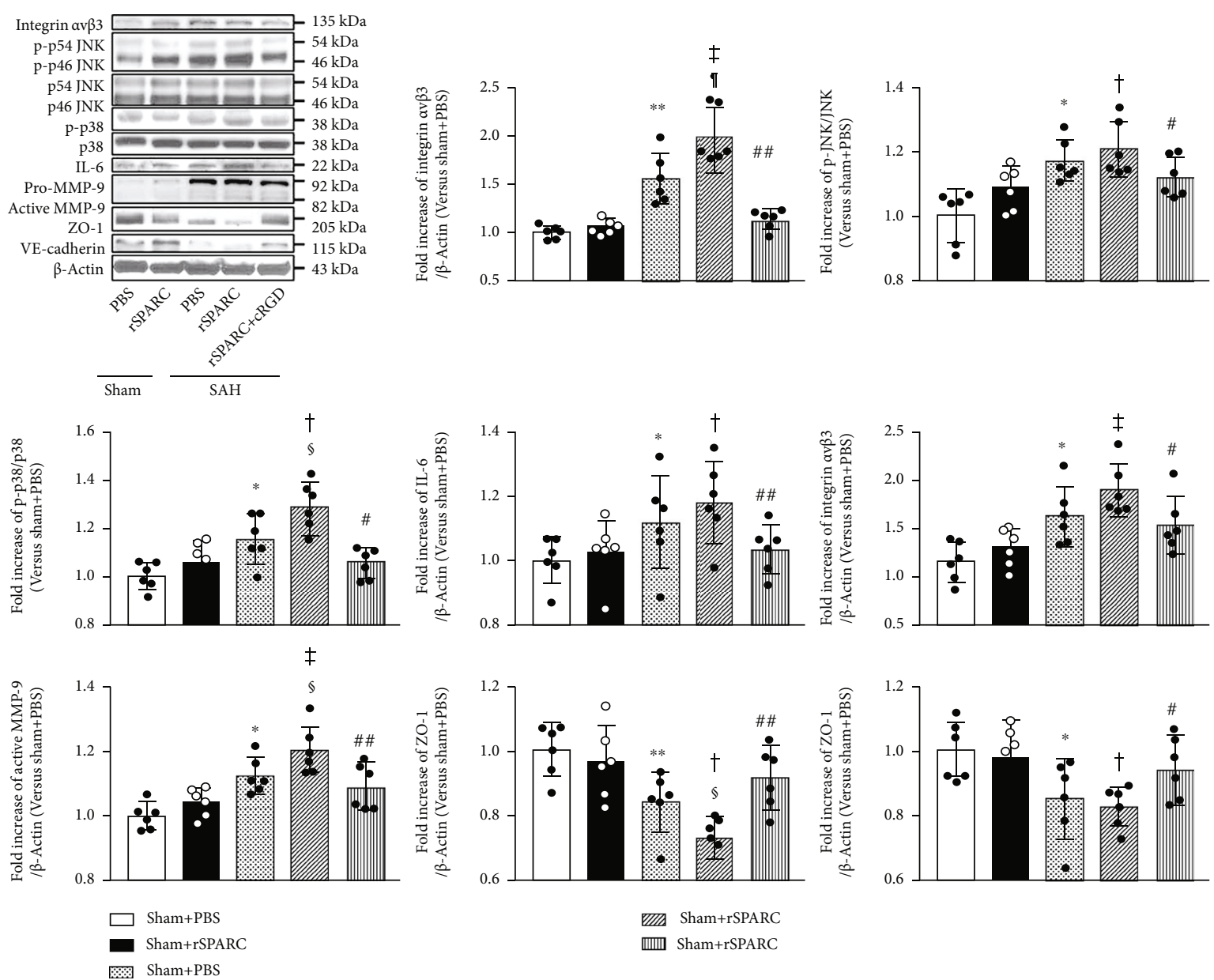

(b)

FIGURE 7: Effects of a recombinant secreted protein acidic and rich in cysteine (rSPARC) and an integrin $\alpha \mathrm{V} \beta 3$ inhibitor at $24 \mathrm{~h}$ after modeling. (a) rSPARC exacerbates modified Garcia's score and inhibition of integrin $\alpha \mathrm{V} \beta 3$ suppresses the effects of rSPARC after SAH ( $n=6$ per group). ${ }^{*} P<0.05$ and ${ }^{* *} P<0.01$. (b) Representative Western blot images and densitometric quantification of expression of phosphorylated p46 and p54 c-Jun N-terminal kinase (p-JNK), phosphorylated p38 (p-p38), interleukin (IL)-6, pro and active matrix metalloproteinase (MMP)-9, ZO-1, and vascular endothelial- (VE-) cadherin. Representative Western blots come from the same samples but multiple membranes. Expression levels of each protein are assessed using $\beta$-actin as an internal control: the levels are expressed as the target protein/ $\beta$-actin, $\mathrm{p}$-p38/p38, or $\mathrm{p}$-JNK/JNK and as a ratio of the average level of the sham + PBS group for normalization, because the levels of p38 and JNK are unchanged among the groups (mean \pm standard deviation; $n=6$ per group). ${ }^{*} P<0.05$ and ${ }^{* *} P<$ 0.01 vs. sham + phosphate-buffered saline (PBS), ${ }^{\dagger} P<0.05$ and ${ }^{\ddagger} P<0.01$ vs. Sham + rSPARC, ${ }^{\circledR} P<0.05$ and ${ }^{5} P<0.01$ vs. SAH + PBS, ${ }^{\#} P<0.05$ and ${ }^{\# \#} P<0.01$ vs. SAH + rSPARC. cRGD: cyclo(-RGDfK): integrin $\alpha \mathrm{V} \beta 3$ inhibitor. 
effects on cerebral blood flow (CBF) and other physiological parameters from postoperation to euthanasia. $\mathrm{CBF}$ and physiological parameters affect brain injury during an acute phase of SAH [60]. To the best of our knowledge, although SPARC has not been reported to affect CBF, blood pressure, and other physiological parameters, it would be worth investigating them. Lastly, the verification of the effects on other animal groups with different age, gender, and genetic background is a subject to be addressed in the future [61]. Although additional experiments need to be performed in the future to solve these limitations, the present study is important for providing a novel potential molecular target SPARC against BBB disruption after SAH.

\section{Conclusion}

This study demonstrated for the first time that blockage of endogenous SPARC prevented post-SAH BBB disruption at least via the inhibition of integrin $\alpha \mathrm{V} \beta 3 / \mathrm{MAPKs} / \mathrm{MMP}$ 9 signaling pathway in rats.

\section{Data Availability}

The data used to support the findings of this study are included within the article.

\section{Conflicts of Interest}

The authors declare that the research was conducted in the absence of any commercial or financial relationships that could be construed as a potential conflict of interest.

\section{Acknowledgments}

The authors acknowledge the technical assistance provided by Desislava Doycheva, Jerry Flores, Marcin Gamdzyk, Helen Huang, and Prativa Sherchan. This article is supported partially by the grants from the National Institutes of Health (grant numbers NS081740 and NS082184) to Dr. Zhang.

\section{Supplementary Materials}

Figure S1: experimental design and animal groups. BWC: brain water content; cRGD: cyclo(-RGDfK): integrin $\alpha \mathrm{V} \beta 3$ inhibitor; IgG: immunoglobulin G; IHC: immunohistochemistry; mAb: monoclonal antibody; PBS: phosphate-buffered saline; SPARC: secreted protein acidic and rich in cysteine; rSPARC: recombinant SPARC; SAH: subarachnoid hemorrhage; siRNA: small interfering ribonucleic acid; Scr siRNA: scrambled siRNA; WB: Western blot. Figure S2: neurobehavioral tests. (a) Modified Garcia's score. (b) Beam balance test. Figure S3: mortality and subarachnoid hemorrhage (SAH) grade. (a) Animal usage and mortality of all experiment groups. (b) Representative basal image of brain in animal models. (c) SAH grade scores of all groups. Data are expressed as median \pm 25 th-75th percentiles. cRGD: cyclo(-RGDfK): integrin $\alpha \mathrm{V} \beta 3$ inhibitor; IgG: immunoglobulin $\mathrm{G}$; $\mathrm{mAb}$ : monoclonal antibody; PBS: phosphate-buffered saline; siRNA: small interfering ribonucleic acid; Scr siRNA: scrambled
siRNA; SPARC: secreted protein acidic and rich in cysteine; rSPARC: recombinant SPARC. Figure S4: the knockdown efficiency of a secreted protein acidic and rich in cysteine (SPARC) small interfering ribonucleic acid (siRNA) at $24 \mathrm{~h}$ after subarachnoid hemorrhage (SAH) induction. (a) Representative Western blot images and densitometric quantification of expressions of SPARC. Expression levels of SPARC are divided by the levels of $\beta$-actin and expressed as a ratio of the average levels of sham models for normalization (mean \pm standard deviation; $n=4$ per group). ${ }^{* *} P<0.01$. (b) Representative immunohistochemical staining of SPARC in the left temporal cortex at $1.0 \mathrm{~mm}$ posterior to the bregma. Nuclei are stained with DAPI (blue). Scale bar $=50 \mu \mathrm{m}$ ( $n=4$ per group). (Supplementary Materials)

\section{References}

[1] E. H. Sage and T. F. Lane, "The biology of SPARC, a protein that modulates cell-matrix interactions," The FASEB Journal, vol. 8, no. 2, pp. 163-173, 1994.

[2] J. van Gijn, R. S. Kerr, and G. J. E. Rinkel, "Subarachnoid haemorrhage," Lancet, vol. 369, no. 9558, pp. 306-318, 2007.

[3] J. I. Suarez, R. W. Tarr, and W. R. Selman, "Aneurysmal subarachnoid hemorrhage," The New England Journal of Medicine, vol. 354, no. 4, pp. 387-396, 2006.

[4] T. Okada, F. Kawakita, H. Nishikawa, F. Nakano, L. Liu, and H. Suzuki, "Selective toll-like receptor 4 antagonists prevent acute blood-brain barrier disruption after subarachnoid hemorrhage in mice," Molecular Neurobiology, vol. 56, no. 2, pp. 976-985, 2019.

[5] T. Okada, B. Enkhjargal, Z. D. Travis et al., "FGF-2 attenuates neuronal apoptosis via FGFR3/PI3k/Akt signaling pathway after subarachnoid hemorrhage," Molecular Neurobiology, vol. 56, no. 12, pp. 8203-8219, 2019.

[6] M. Sabri, E. Lass, and R. L. Macdonald, "Early brain injury: a common mechanism in subarachnoid hemorrhage and global cerebral ischemia," Stroke research and treatment, vol. 2013, Article ID 394036, 2013.

[7] H. Suzuki, "What is early brain injury?," Translational Stroke Research, vol. 6, no. 1, pp. 1-3, 2015.

[8] M. Sabri, J. Ai, E. Lass, J. D’abbondanza, and R. L. Macdonald, "Genetic elimination of eNOS reduces secondary complications of experimental subarachnoid hemorrhage," Journal of cerebral blood flow and metabolism : official journal of the International Society of Cerebral Blood Flow and Metabolism, vol. 33, no. 7, pp. 1008-1014, 2013.

[9] B. P. Lucke-Wold, A. F. Logsdon, B. Manoranjan et al., "Aneurysmal subarachnoid hemorrhage and neuroinflammation: a comprehensive review," International Journal of Molecular Sciences, vol. 17, no. 4, p. 497, 2016.

[10] H. Kanamaru and H. Suzuki, "Potential therapeutic molecular targets for blood-brain barrier disruption after subarachnoid hemorrhage," Neural Regeneration Research, vol. 14, no. 7, pp. 1138-1143, 2019.

[11] H. Suzuki, H. Nishikawa, and F. Kawakita, "Matricellular proteins as possible biomarkers for early brain injury after aneurysmal subarachnoid hemorrhage," Neural Regeneration Research, vol. 13, no. 7, pp. 1175-1178, 2018.

[12] F. Kawakita, H. Kanamaru, R. Asada, and H. Suzuki, "Potential roles of matricellular proteins in stroke," Experimental Neurology, vol. 322, article 113057, 2019. 
[13] S. Alkabie, J. Basivireddy, L. Zhou, J. Roskams, P. Rieckmann, and J. A. Quandt, "SPARC expression by cerebral microvascular endothelial cells in vitro and its influence on blood-brain barrier properties," Journal of Neuroinflammation, vol. 13, no. 1, pp. 1-17, 2016.

[14] A. J. Vincent, P. W. Lau, and A. J. Roskams, "SPARC is expressed by macroglia and microglia in the developing and mature nervous system," Developmental Dynamics, vol. 237, no. 5, pp. 1449-1462, 2008.

[15] D. B. Mendis, G. O. Ivy, and I. R. Brown, "SPARC/osteonectin mRNA is induced in blood vessels following injury to the adult rat cerebral cortex," Neurochemical Research, vol. 23, no. 8, pp. 1117-1123, 1998.

[16] Y. Takada, X. Ye, and S. Simon, "The integrins," Genome Biology, vol. 8, no. 5, p. 215, 2007.

[17] Y. H. Soung, J. L. Clifford, and J. Chung, "Crosstalk between integrin and receptor tyrosine kinase signaling in breast carcinoma progression," BMB Reports, vol. 43, no. 5, pp. 311-318, 2010.

[18] L. Liu, F. Kawakita, M. Fujimoto et al., "Role of periostin in early brain injury after subarachnoid hemorrhage in mice," Stroke, vol. 48, no. 4, pp. 1108-1111, 2017.

[19] H. Suzuki, M. Fujimoto, M. Shiba et al., "The role of matricellular proteins in brain edema after subarachnoid hemorrhage," Acta Neurochirurgica. Supplement, vol. 121, pp. 151-156, 2016.

[20] H. C. Chong, C. K. Tan, R.-L. Huang, and N. S. Tan, "Matricellular proteins: a sticky affair with cancers," Journal of Oncology, vol. 2012, Article ID 351089, 2012.

[21] L. B. Rivera, A. D. Bradshaw, and R. A. Brekken, "The regulatory function of SPARC in vascular biology," Cellular and Molecular Life Sciences, vol. 68, no. 19, pp. 3165-3173, 2011.

[22] S. S. Smyth and C. Patterson, "Tiny dancers: the integringrowth factor nexus in angiogenic signaling," Journal of Cell Biology, vol. 158, no. 1, pp. 17-21, 2002.

[23] H. Suzuki, Y. Hasegawa, K. Kanamaru, and J. H. Zhang, "Mechanisms of osteopontin-induced stabilization of bloodbrain barrier disruption after subarachnoid hemorrhage in rats," Stroke, vol. 41, no. 8, pp. 1783-1790, 2010.

[24] M. Fujimoto, M. Shiba, F. Kawakita et al., "Effects of tenascinC knockout on cerebral vasospasm after experimental subarachnoid hemorrhage in mice," Molecular Neurobiology, vol. 55, no. 3, pp. 1951-1958, 2018.

[25] D. N. Edwards and G. J. Bix, "Roles of blood-brain barrier integrins and extracellular matrix in stroke," American Journal of Physiology-Cell Physiology, vol. 316, no. 2, pp. C252-C263, 2019.

[26] F. A. Sehba, G. Mostafa, J. Knopman, V. Friedrich, and J. B. Bederson, "Acute alterations in microvascular basal lamina after subarachnoid hemorrhage," Journal of Neurosurgery, vol. 101, no. 4, pp. 633-640, 2004.

[27] T. Sugawara, R. Ayer, V. Jadhav, and J. H. Zhang, "A new grading system evaluating bleeding scale in filament perforation subarachnoid hemorrhage rat model," Journal of Neuroscience Methods, vol. 167, no. 2, pp. 327-334, 2008.

[28] M. T. Sweetwyne, R. A. Brekken, G. Workman et al., "Functional analysis of the matricellular protein SPARC with novel monoclonal antibodies," Journal of Histochemistry and Cytochemistry, vol. 52, no. 6, pp. 723-733, 2004.

[29] J. Westerhout, B. Ploeger, J. Smeets, M. Danhof, and E. C. M. de Lange, "Physiologically based pharmacokinetic modeling to investigate regional brain distribution kinetics in rats," The AAPS Journal, vol. 14, no. 3, pp. 543-553, 2012.

[30] S. Talegaonkar and P. R. Mishra, "Intranasal delivery : an approach to bypass the blood brain barrier," Indian Journal of Pharmacology, vol. 36, no. 3, pp. 140-147, 2004.

[31] J. Šimeček, J. Notni, T. G. Kapp, H. Kessler, and H. J. Wester, "Benefits of NOPO as chelator in gallium-68 peptides, exemplified by preclinical characterization of $68 \mathrm{Ga}-\mathrm{NOPO}-$ c(RGDfK)," Molecular Pharmaceutics, vol. 11, no. 5, pp. 1687-1695, 2014.

[32] C. M. Verkeste, B. F. M. Slangen, M. L. Dubelaar, B. K. Van Kreel, and L. L. H. Peeters, "Mechanism of volume adaptation in the awake early pregnant rat," American Journal of Physiology-Heart and Circulatory Physiology, vol. 274, no. 5, pp. H1662-H1666, 1998.

[33] T. Abumiya, J. Lucero, J. H. Heo et al., "Activated microvessels express vascular endothelial growth factor and integrin alpha(v)beta3 during focal cerebral ischemia," Journal of cerebral blood flow and metabolism : official journal of the International Society of Cerebral Blood Flow and Metabolism, vol. 19, no. 9, pp. 1038-1050, 1999.

[34] E. Peixoto, C. Atorrasagasti, J. B. Aquino et al., "SPARC (secreted protein acidic and rich in cysteine) knockdown protects mice from acute liver injury by reducing vascular endothelial cell damage," Gene Therapy, vol. 22, no. 1, pp. 9-19, 2015.

[35] E. V. Jones and D. S. Bouvier, "Astrocyte-secreted matricellular proteins in CNS remodelling during development and disease," Neural Plasticity, vol. 2014, Article ID 321209, 2014.

[36] K. Jacob, M. Webber, D. Benayahu, and H. K. Kleinman, "Osteonectin promotes prostate cancer cell migration and invasion: a possible mechanism for metastasis to bone," Cancer Research, vol. 59, no. 17, pp. 4453-4457, 1999.

[37] C. Gilles, J. A. Bassuk, H. Pulyaeva, E. H. Sage, J. M. Foidart, and E. W. Thompson, "SPARC/osteonectin induces matrix metalloproteinase 2 activation in human breast cancer cell lines," Cancer Research, vol. 58, no. 23, pp. 5529-5536, 1998.

[38] P. M. Tremble, T. F. Lane, E. H. Sage, and Z. Werb, "SPARC, a secreted protein associated with morphogenesis and tissue remodeling, induces expression of metalloproteinases in fibroblasts through a novel extracellular matrix-dependent pathway," Journal of Cell Biology, vol. 121, no. 6, pp. 1433-1444, 1993.

[39] S. E. Goldblum, X. Ding, S. E. Funk, and E. H. Sage, "SPARC (secreted protein acidic and rich in cysteine) regulates endothelial cell shape and barrier function," Proceedings of the National Academy of Sciences of the United States of America, vol. 91, no. 8, pp. 3448-3452, 1994.

[40] T. Tilling, C. Engelbertz, S. Decker, D. Korte, S. Hüwel, and H.J. J. Galla, "Expression and adhesive properties of basement membrane proteins in cerebral capillary endothelial cell cultures," Cell and Tissue Research, vol. 310, no. 1, pp. 19-29, 2002.

[41] B. Srinivasan, A. R. Kolli, M. B. Esch, H. E. Abaci, M. L. Shuler, and J. J. Hickman, "TEER measurement techniques for in vitro barrier model systems," Journal of Laboratory Automation, vol. 20, no. 2, pp. 107-126, 2015.

[42] H. Nishikawa and H. Suzuki, "Implications of periostin in the development of subarachnoid hemorrhage-induced brain injuries," Neural Regeneration Research, vol. 12, no. 12, p. 1982, 2017. 
[43] M. A. Petty and E. H. Lo, "Junctional complexes of the bloodbrain barrier: permeability changes in neuroinflammation," Progress in Neurobiology, vol. 68, no. 5, pp. 311-323, 2002.

[44] H. Suzuki and F. Kawakita, "Tenascin-C in aneurysmal subarachnoid hemorrhage: deleterious or protective?" Neural Regeneration Research, vol. 11, no. 2, pp. 230-231, 2016.

[45] T. Okada, H. Suzuki, Z. D. Travis, and J. H. Zhang, "The stroke-induced blood-brain barrier disruption: current progress of inspection technique, mechanism, and therapeutic target," Current Neuropharmacology, vol. 18, no. 12, pp. 11871212, 2020.

[46] M. Fujimoto, M. Shiba, F. Kawakita et al., "Deficiency of tenascin-C and attenuation of blood-brain barrier disruption following experimental subarachnoid hemorrhage in mice," Journal of Neurosurgery, vol. 124, no. 6, pp. 1693-1702, 2016.

[47] Y. Yang, E. Y. Estrada, J. F. Thompson, W. Liu, and G. A. Rosenberg, "Matrix metalloproteinase-mediated disruption of tight junction proteins in cerebral vessels is reversed by synthetic matrix metalloproteinase inhibitor in focal ischemia in rat," Journal of Cerebral Blood Flow and Metabolism, vol. 27, no. 4, pp. 697-709, 2007.

[48] Y. Shigemori, Y. Katayama, T. Mori, T. Maeda, and T. Kawamata, "Matrix metalloproteinase-9 is associated with blood-brain barrier opening and brain edema formation after cortical contusion in rats," Acta Neurochirurgica. Supplement, vol. 96, pp. 130-133, 2006.

[49] H. Suzuki, R. Ayer, T. Sugawara et al., "Protective effects of recombinant osteopontin on early brain injury after subarachnoid hemorrhage in rats," Critical Care Medicine, vol. 38, no. 2, pp. 612-618, 2010.

[50] S. Feiler, N. Plesnila, S. C. Thal, S. Zausinger, and K. Schöller, "Contribution of matrix metalloproteinase-9 to cerebral edema and functional outcome following experimental subarachnoid hemorrhage," Cerebrovascular Diseases, vol. 32, no. 3, pp. 289-295, 2011.

[51] P. Sherchan, T. Lekic, H. Suzuki et al., "Minocycline improves functional outcomes, memory deficits, and histopathology after endovascular perforation-induced subarachnoid hemorrhage in rats," Journal of Neurotrauma, vol. 28, no. 12, pp. 2503-2512, 2011.

[52] M. Shiba and H. Suzuki, "Lessons from tenascin-C knockout mice and potential clinical application to subarachnoid hemorrhage," Neural Regeneration Research, vol. 14, no. 2, pp. 262-264, 2019.

[53] H. Suzuki, M. Fujimoto, F. Kawakita et al., "Tenascin-C in brain injuries and edema after subarachnoid hemorrhage: findings from basic and clinical studies," Journal of Neuroscience Research, vol. 98, no. 1, pp. 42-56, 2020.

[54] T. Okada and H. Suzuki, "Mechanisms of neuroinflammation and inflammatory mediators involved in brain injury following subarachnoid hemorrhage," Histology and Histopathology, vol. 35, no. 7, pp. 623-636, 2020.

[55] T. Okada and H. Suzuki, "Toll-like receptor 4 as a possible therapeutic target for delayed brain injuries after aneurysmal subarachnoid hemorrhage," Neural Regeneration Research, vol. 12, no. 2, pp. 193-196, 2017.

[56] D. Chen, X. Wei, J. Guan et al., "Inhibition of c-Jun N-terminal kinase prevents blood-brain barrier disruption and normalizes the expression of tight junction proteins clautin- 5 and $\mathrm{ZO}-1$ in a rat model of subarachnoid hemorrhage," Acta Neurochirurgica, vol. 154, no. 8, pp. 1469-1476, 2012.
[57] C. Kupprion, K. Motamed, and E. H. Sage, "SPARC (BM-40, osteonectin) inhibits the mitogenic effect of vascular endothelial growth factor on microvascular endothelial cells," Journal of Biological Chemistry, vol. 273, no. 45, pp. 29635-29640, 1998.

[58] L. Liu, M. Fujimoto, F. Kawakita et al., “Anti-vascular endothelial growth factor treatment suppresses early brain injury after subarachnoid hemorrhage in mice," Molecular Neurobiology, vol. 53, no. 7, pp. 4529-4538, 2016.

[59] T. Sugawara, R. Ayer, V. Jadhav, W. Chen, T. Tsubokawa, and J. H. Zhang, "Simvastatin attenuation of cerebral vasospasm after subarachnoid hemorrhage in rats via increased phosphorylation of Akt and endothelial nitric oxide synthase," Journal of Neuroscience Research, vol. 86, no. 16, pp. 36353643, 2008.

[60] F. A. Sehba, J. Hou, R. M. Pluta, and J. H. Zhang, "The importance of early brain injury after subarachnoid hemorrhage," Progress in Neurobiology, vol. 97, no. 1, pp. 14-37, 2012. 